\title{
Efficacy and Safety of Proton Beam Therapy for Primary Optic Nerve Sheath Meningioma
}

\author{
Rabih Hage (D) \\ Claire Alapetite ${ }^{2}$ \\ Hervé Brisse $\mathbb{D}^{3,4}$ \\ Kevin Zuber ${ }^{5}$ \\ Augustin Lecler $\mathbb{I D}^{6}$ \\ Guillaume Lot $^{7}$ \\ Caroline Le Guerinel $^{7}$ \\ Catherine Vignal- \\ Clermont (D) \\ Herve Boissonnet ${ }^{7}$ \\ 'Neuro-ophthalmology \\ Department, Hôpital Fondation A. de \\ Rothschild, Paris, France; ${ }^{2}$ Radiation \\ Oncology Department, and Proton \\ Center Institut Curie, Paris-Orsay, \\ France; ${ }^{3}$ Imaging Department, Institut \\ Curie, Paris, France; ${ }^{4}$ Université des \\ Sciences et Lettres, Paris, France; \\ ${ }^{5}$ Department of Statistics and Clinical \\ Research, Hôpital Fondation A. de \\ Rothschild, Paris, France; ${ }^{6}$ Radiology \\ Department, Hôpital Fondation A. de \\ Rothschild, Paris, France; ${ }^{7}$ Neurosurgery \\ Department, Hôpital Fondation A. de \\ Rothschild, Paris, France
}

Purpose: Management of optic nerve sheath meningiomas (ONSM) remains challenging. Photon radiation therapy (PhRT) is the most common treatment for sight-threatening ONSM. Proton beam therapy (PBT) is less commonly used because it is more expensive and because there are questions about its efficacy specifically in relation to ONSM. PBT has the theoretical advantage of reducing radiation exposure to adjacent structures. We report the visual outcome of patients with primary ONSM managed at the Fondation Ophtalmologique Adolphe de Rothschild, Paris, France, and treated with PBT at the Centre de Protonthérapie, Institut Curie, Orsay, France.

Methods: We conducted a retrospective review of all patients with primary ONSM who received PBT (either by itself or following surgery) between January 2006 and January 2019. Neuro-ophthalmic examinations were performed at presentation and after radiotherapy, and, when applicable, after surgery. Meningiomas were measured at the time of diagnosis and at each follow-up MRI examination.

Results: Sixty patients (50 women, 10 men; mean age, $45.2 \pm 11.1 \mathrm{y}$ ) were included, of whom 29 underwent surgery. At presentation, $52(87 \%)$ of them had decreased vision (average visual acuity: $0.6 \log M A R)$. Fundus examination showed optic disc swelling ( $n=27 ; 46.5 \%)$, optic disc pallor $(n=22 ; 37.9 \%)$, optic disc cupping $(n=2 ; 3.4 \%)$, opto-ciliary shunt $(n=8$; $13.8 \%)$, or choroidal folds $(n=5 ; 8.6 \%)$. Otherwise, it was unremarkable $(n=7 ; 12.1 \%)$. After treatment, visual function was stable overall. Fundus examination showed pallor $(n=47$; $83.9 \%)$, swelling $(n=3 ; 5.4 \%)$, or cupping $(n=2 ; 3.4 \%)$ of the optic disc, or was unremarkable $(\mathrm{n}=5 ; 8.9 \%)$. The visual field of 8 patients worsened, while 3 developed asymptomatic retinal hemorrhages. Tumor shrunk significantly in 8 patients at 1 year after PBT and remained stable in size in all others. Patients with opto-ciliary shunts had significantly worse visual outcome than other patients. Retinal abnormalities were observed in 11 patients during follow-up.

Conclusion: PBT alone or in association with surgery appears to be a safe and efficient treatment for ONSM, reducing the tumor size and stabilizing visual function. The risk of developing radiation retinopathy seems to be higher when patients had upfront surgery.

Keywords: optic nerve sheath meningioma, ONSM, proton beam therapy, PBT, compressive optic neuropathy, brain tumor, visual loss, opto-ciliary shunt, radiation retinopathy, RON

\section{Introduction}

Primary optic nerve sheath meningiomas (ONSM) are benign tumors originating from the meningothelial cells surrounding the intraorbital or the intracanalicular portion of the optic nerve (ON). They account for approximately $2 \%$ of all orbital tumors and represent the most common neoplastic cause of compressive optic
Correspondence: Rabih Hage

Tel/Fax +33148036322

Email rhage@for.paris 
neuropathy, after pituitary adenoma. ONSM are significantly more common in middle-aged women (61-84\%) and most of them are unilateral $(95 \%){ }^{1}$

ONSM grow slowly and progressively and can remain asymptomatic for several years before chronic compression of the optic nerve fibers causes decreased vision or visual field loss. At present, management of ONSM is challenging, but normally involves monitoring of visual function. Treatment must be considered when there is evidence of $\mathrm{ON}$ damage with risk of visual loss. Possible interventions include surgery or radiotherapy. However, neither of these is systematic since they are invasive and can lead to further visual loss. ${ }^{2,3}$

There is high risk of iatrogenic injury to the $\mathrm{ON}$ with surgery because of the close relationship between the tumor and ON blood supply. It is not feasible to dissect the tumor itself, which would result in $\mathrm{ON}$ ischemia and severe loss of vision. The goal of surgery is to make room for the $\mathrm{ON}$ as it is squeezed by the tumor (eg, as it goes through the optic canal). Unfortunately, up to $56 \%$ of ONSM patients who undergo orbital surgery experience a decline in visual outcome. ${ }^{2}$ Thus, surgery is considered in cases of advanced disease with intracranial component, if there is a risk of extension to the contralateral ON, or for cosmetic reasons in patients with severe proptosis. ${ }^{4}$

When there is decline in visual function but no indication for surgery, radiation therapy is considered. Various techniques of photon radiation therapy (PhRT) have been used: conformal radiotherapy, intensity modulated radiation therapy, fractionated stereotactic radiotherapy, and stereotactic radiosurgery. ${ }^{3,5-7}$ Patients with ONSM who receive radiotherapy are at risk of developing radiation optic neuropathy (RON), which can precipitate visual loss. ${ }^{8}$ Stereotactic fractionated photon radiotherapy (SRT) based on 3-dimensional treatment planning has shown encouraging results alone and in association with conservative surgery. SRT for ONSM typically delivers a total of 50-54 Gy, usually divided in 25-28 fractions distributed daily over the course of 5-6 weeks. It allows control of tumor growth and stabilization of visual loss in most cases. ${ }^{4}$ However, gradual deterioration in visual and endocrine function - related to pituitary irradiation - has been observed 3 years after completion of therapy. ${ }^{5,6}$ Radiation therapy dose and volume prescription must be carefully balanced to avoid undertreating the tumor on the one hand and overpassing optic pathway tolerance on the other. ${ }^{7}$

Proton beam therapy (PBT) is a promising alternative to PhRT. As opposed to photons, proton beams have a finite range and a narrow lateral penumbra that theoretically allow reduced exposure of normal tissues beyond the target volume. Depending on the extension of the tumor, optimized protection of ipsilateral and contralateral ONs and retina, chiasm, and pituitary gland may be obtained by PBT. Depending on tumor, therefore, PBT can target a tumor without having "spill-over" effect on these other structures. Total dose fractionation and 3-dimensional planning also apply to protons to minimize the risk of radiation-related morbidity. PBT remains a scarce resource with limited access. The cost and complexity of this technology have limited its development. Here, we report a retrospective analysis of 60 consecutive cases of primary ONSM treated with PBT.

\section{Methods}

\section{Inclusion Criteria and Patient Cohort}

Patients included in this retrospective study were adults $(>18$ years old) with a diagnosis of primary ONSM seen between January 2006 and January 2019 at the Fondation Ophtalmologique Adolphe de Rothschild in Paris, France, and who received PBT at the Institut Curie in Orsay, France.

The diagnosis of ONSM was based on MRI findings or on histological specimens from operated patients. Primary ONSM were defined as meningiomas originating from the ON sheath. Orbital and cerebral meningioma extending into the orbits and the $\mathrm{ON}$ sheath were not included in the analysis. PBT was given alone or following decompression surgery. Routine management of ONSM is further described in the following sections. The Institutional Review Boards of both institutions waived the need for written informed consent and approved the study.

\section{MRI Analysis}

MRI examinations were retrieved from the PACS (Picture Archiving and Communication System) of both institutions (Carestream Healthcare) and reviewed by two neuroradiologists and one neurosurgeon. Decisions were made by consensus.

The retrieved MRI examinations were performed on different MRI units and at different institutions, but all of them included 2D or 3D T1- and T2-weighted sequences, and gadolinium-based contrast-enhanced T1-weighted sequences with fat suppression in at least two orthogonal acquisition planes.

MRI analyses included evaluation of the tumor (size, shape, and signal) at diagnosis, post-surgery (when 
applicable), and post-PBT. In patients who underwent surgery, post-surgical MRI was used as the reference pre-PBT MRI when radiotherapy was scheduled within the 3-4 months following surgery. In cases with longer duration between surgery and PBT, MRI was repeated before the beginning of PBT. Post-PBT, the reference post-treatment MRI used for comparison was the one performed 6-12 months after the end of PBT.

Tumors were measured and graded according to their location and pattern of growth using Schick's MRI classification (Figure 1). ${ }^{9}$

Changes in tumor size were assessed through a twodimension approach. The RECIST (Response Evaluation Criteria In Solid Tumors) analysis, based on the single largest diameter of the tumor, was considered inappropriate for ONSM, which frequently remain unchanged in their longest axis. The following measurements were made on each MRI: 1) D1 diameter, defined as the longest diameter parallel to the axis of the $\mathrm{ON}$, and 2) D2 diameter, defined as the longest diameter perpendicular to D1 on the same sequence (or, if higher, the longest diameter on coronal sequence). The product of these orthogonal diameters was used to assess tumor surface.
According to the WHO 2D-response criteria, partial response (PR) was defined as a decrease of at least $50 \%$ of tumor surface and progressive disease (PD) as an increase of at least $25 \%$. In intermediate cases, tumor size was deemed stable (ST) ${ }^{10}$

\section{Management}

All cases were discussed in multidisciplinary meetings that always included at least one neurosurgeon, one neuroophthalmologist, one neuroradiologist and one radiation oncologist. The following management options were discussed either as monotherapy or in various combinations: steroids, surgery, radiotherapy, and monitoring. When diagnosis by MR imaging was uncertain, surgical biopsy was performed for pathology examination and hormone receptors assay. ${ }^{11}$

- IV or oral steroid treatment was discussed as a firstline treatment in cases of ON head swelling or severe visual loss.

- When radiotherapy alone was thought insufficient because of compression in the optic canal, intracranial extension, or risk of contralateral spread, surgery
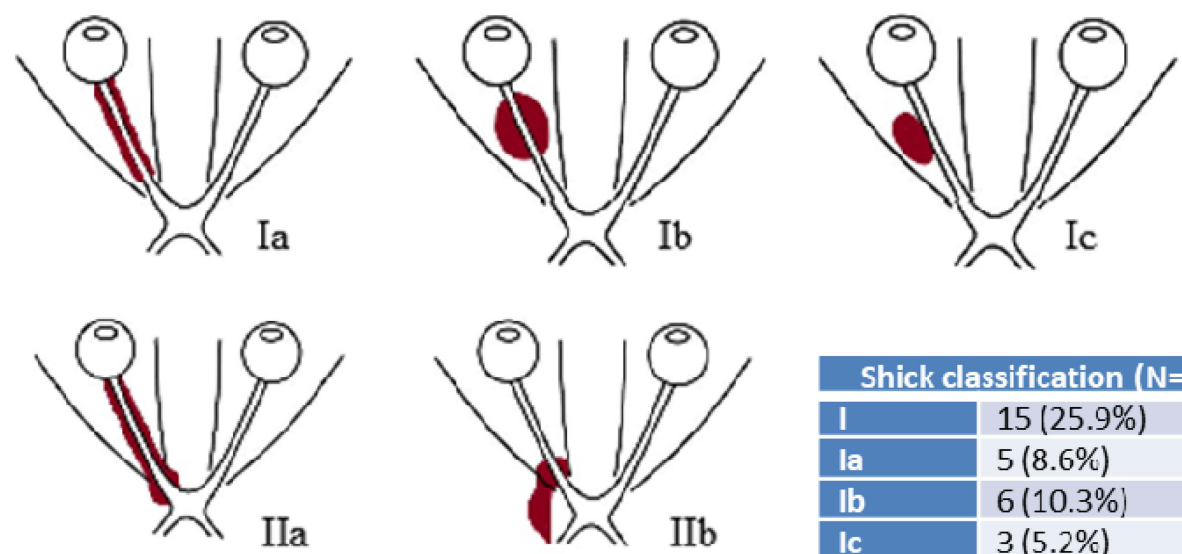

\begin{tabular}{|l|l|}
\multicolumn{2}{|c|}{ Shick classification (N=58) } \\
\hline I & $15(25.9 \%)$ \\
\hline la & $5(8.6 \%)$ \\
\hline Ib & $6(10.3 \%)$ \\
\hline Ic & $3(5.2 \%)$ \\
\hline Ila & $18(31 \%)$ \\
\hline IIb & $3(5.2 \%)$ \\
\hline III & $1(1,7 \%)$ \\
\hline IIIb & $7(12.1 \%)$ \\
\hline
\end{tabular}
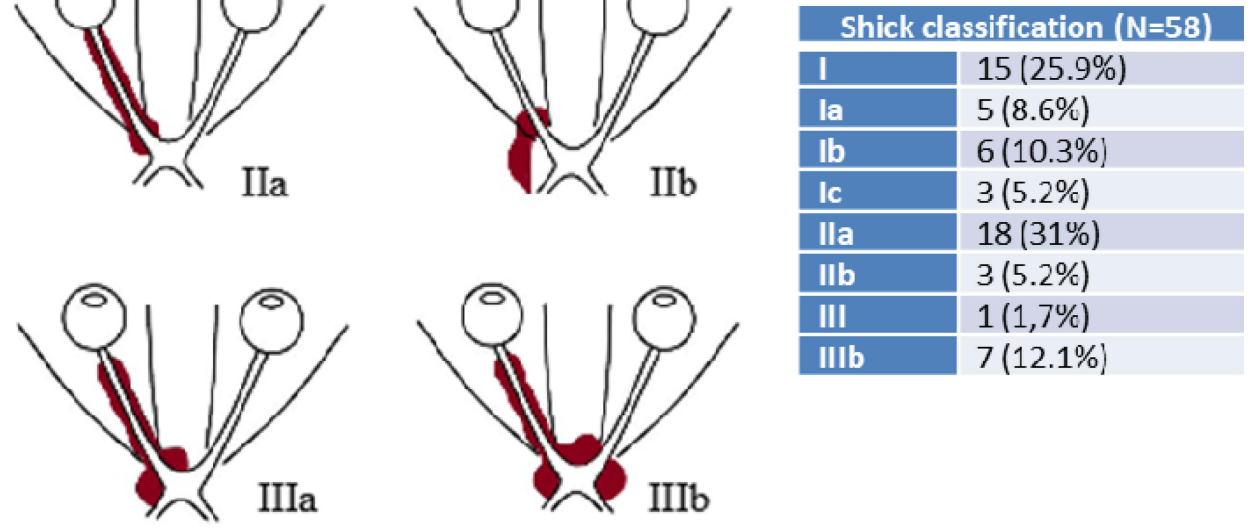

Figure I Types and subtypes of ONSM according to their location, adapted from Schick et al. ${ }^{9}$ Upper: Type I, purely intraorbital lesions: Type la: flat tumor extension around the optic nerve; Type lb: bulbiform mass around the optic nerve; Type Ic: exophytic tumor on the optic nerve. Center: Type II, intraorbital ONSM with extension through the optic canal or superior orbital fissure: Type lla: intraorbital tumor with growth through the optic canal; Type Ilb: tumors of the apex, superior orbital fissure, or cavernous. Lower: Type III, intraorbital lesions with widespread intracranial tumor extension: Type IIla: intracranial extension to the chiasm; Type IIlb: widespread intracranial extension to the chiasm, contralateral optic nerve, and planum sphenoidale. 
for decompression or partial resection was discussed. Surgery consisted of one of the following 6 interventions: limited biopsy of the tumor when diagnosis was doubtful, debulking or orbitotomy in case of severe orbital compression, ON sheath fenestration, or optic canal opening.

- Before October 2010, PBT used a fixed horizontal beam of $201 \mathrm{MeV}$, accelerated by a synchrocyclotron. After October 2010, PBT used a fixed horizontal beam of $230 \mathrm{MeV}$, accelerated by a cyclotron (PROTEUS Plus, IBA, Louvain-laNeuve, Belgium). All treatments were delivered using the passive scattering technique. Median total dose prescribed was 52.2 GyRBE. Median prescribed dose per fraction was 1.8 GyRBE. Five fractions were delivered each week (one per day). In cases with previous surgery and severe visual impact, slightly lower total dose and dose per fraction (1.7 GyRBE) were prescribed. Prescribed dose constraint to the ipsilateral retina was 50 GyRBE in most cases. Between 1996 and 2008, treatment plans were generated using the ISIS 3D treatment planning system (Technology Diffusion). Starting 2009, ISOgray treatment planning system (DOSIsoft, Cachan, France) was used. Ballistic approach used typically 4 beams ( 3 or 4 in few cases).

\section{Ophthalmic Assessment}

All patients were examined by a trained neuroophthalmologist at the time of diagnosis, within 3 months following surgery (when performed), and pre- and postPBT. Ophthalmic examination included visual acuity measurement, slit-lamp and fundus examination, visual field (VF) testing with Humphrey perimetry (when visual acuity was better than $0.7 \operatorname{logMAR}$, ie, 20/100), Goldmann perimetry (when visual acuity was $0.7 \log$ MAR or lower), and optical coherence tomography (OCT) of the ON. OCT was performed using Spectralis Spectral-Domain OCT (Heidelberg Engineering, Heidelberg, Germany). When both eyes were affected, the one that had the better visual acuity was included in the analysis. The following data were recorded: best-corrected visual acuity (converted to $\log$ MAR), presence of exophthalmos, anomalies of the ON (edema, pallor or excavation), mean deviation (MD) of the automatic Humphrey visual field, and average retinal nerve fiber layer (RNFL) thickness. When visual acuity was not measurable (lower than "seeing hand motions", which is the equivalent of "2.3 $\log$ MAR"), it was noted as
“2.5 logMAR". Visual field defect was classified as "central" when there was involvement of the central $10^{\circ}$ and as "peripheral" when defect was outside the central $10^{\circ}$. Preand post-treatment visual field examinations were compared and post-treatment visual field was interpreted as "improved" when there was no longer central or peripheral visual field defects, as "stable" when visual field defects were similar to pretreatment visual field, or as "worsened" when a central or peripheral visual field defect appeared after treatment. This analysis was performed by 2 trained neuro-ophthalmologists. In cases of discrepancy, consensus was reached after discussion.

\section{Statistics}

Data analysis was performed using $\mathrm{R}$ statistical software ( $\mathrm{R}$ core team, 2013, R Foundation for Statistical Computing, Vienna, Austria). Descriptive statistics were used for presenting the demographic characteristics of the cohort. Visual acuity and visual field measurement were compared pre- and post-surgery, and pre- and post-PBT using Wilcoxon signed-rank test. The prognostic values of fundus findings on visual outcome were tested (MannWhitney $U$ test) and the correlation between initial and final visual acuity and visual fields was tested (Spearman) as the data were not normally distributed. P-values $<0.05$ were considered significant.

\section{Results \\ Patients' Characteristics}

Sixty patients, 50 women and 10 men, were included. Median age at diagnosis was 45.2 years (34.1-56.3). Patients' characteristics are summarized in Table 1. Decreased vision was the main complaint and was reported by $87 \%(n=52)$ of the patients at presentation. Other symptoms were orbital pain, exophthalmos, diplopia, and eye redness, all of which could be associated with decreased vision. In 5 patients, ONSM were part of meningiomatosis, which is defined by the presence of at least 2 simultaneous lesions at different intracranial locations without an association with neurofibromatosis type 2 . None of the patients had a history of neurofibromatosis or acoustic neuroma. Diagnosis was made on average 16.3 months (0-240) (InterQuartile Range $(\mathrm{IQR})=17)$ after the first symptoms. Delay in diagnosis was not correlated with initial visual acuity $(\mathrm{r}=0.015$, Spearman correlation) or visual field MD ( $\mathrm{r}=0.02$, Spearman correlation). 
Table I Patient Characteristics $(\mathrm{N}=60)$

\begin{tabular}{|c|c|}
\hline \multicolumn{2}{|l|}{ Gender } \\
\hline - Male & $10(16.6 \%)$ \\
\hline - Female & $50(83.3 \%)$ \\
\hline Mean age at diagnosis, years & $45.2 \pm 11.1$ \\
\hline $\begin{array}{l}\text { Median duration of symptoms before } \\
\text { diagnosis, months }\end{array}$ & $7.5[0-240]$ \\
\hline Hormonal treatment in women, number & 21 (42\%) (data unavailable in 2$)$ \\
\hline \multicolumn{2}{|l|}{ Clinical presentation } \\
\hline - Visual loss & $52(86.6 \%)$ \\
\hline - Progressive & $33(55 \%)$ \\
\hline • Sudden & $15(25 \%)$ \\
\hline $\begin{array}{l}\bullet \text { Incidentally noticed on routine } \\
\text { eye examination }\end{array}$ & $4(6.6 \%)$ \\
\hline - Orbital pain & $15(25 \%)$ \\
\hline - Exophthalmos & $21(35 \%)$ \\
\hline - Eye redness & 7 (1 $1.6 \%)$ \\
\hline - Meningiomatosis & $5(8.3 \%)$ \\
\hline - Headaches/Eye pain & $15(25 \%)$ \\
\hline - Diplopia & $10(16.6 \%)$ \\
\hline \multicolumn{2}{|l|}{ Neurosurgery before Proton Beam } \\
\hline \multicolumn{2}{|l|}{ Therapy (PBT) } \\
\hline - None & 29 (one had surgery after PBT) \\
\hline - Biopsy & 6 \\
\hline - Biopsy + Resection & 6 \\
\hline - Intracranial extension resection & 15 \\
\hline - Other surgeries & 3 \\
\hline
\end{tabular}

Visual acuity was 0.6 $\log \mathrm{MAR}$ on average $(\mathrm{IQR}=1.0)$. Initial fundus examination data were available in 58 patients and were normal in 7. Fundus abnormalities included swelling, pallor or cupping of the ON head, optociliary shunt and choroidal folds (Table 2). Of the 60 included patients, 27 had visual acuity that allowed automatized visual field examination. In these patients, mean deviation was $-13.4(\mathrm{IQR}=9.8)$ and foveal threshold was 29.4 (IQR=5.5). RNFL thickness was measured in only 34 patients and was $119.5 \mu$ on average $(\mathrm{IQR}=63.8)$. Visual field examination (including static and kinetic) showed involvement of the peripheral visual field in 41 patients and involvement of the central $10^{\circ}$ in 32 patients. Three patients had vision that did not allow visual field examination (light perception or hand motion).

\section{Surgery}

Twenty-eight patients had upfront surgery before PBT. Surgery consisted of biopsy of the tumor for pathological analysis in 6 patients. Twenty-one patients had debulking surgery, 4 orbitotomy, $11 \mathrm{ON}$ sheath fenestration, and 21
Table 2 Fundus Examination

\begin{tabular}{|c|c|c|c|}
\hline & $\begin{array}{l}\text { Initial } \\
\text { Examination }\end{array}$ & Pre-PBT & Final \\
\hline & $N=58$ & $N=55$ & $N=58$ \\
\hline $\begin{array}{l}\text { ON head } \\
\text { - Swelling } \\
\text { - Pallor } \\
\text { - Cupping }\end{array}$ & $\begin{array}{l}27(46.5 \%) \\
22(37.9 \%) \\
2(3.4 \%)\end{array}$ & $\begin{array}{l}19(36.5 \%) \\
32(61.5 \%) \\
2(3.8 \%)\end{array}$ & $\begin{array}{l}3(5.4 \%) \\
47(83.9 \%) \\
2\end{array}$ \\
\hline $\begin{array}{l}\text { Opto-ciliary } \\
\text { shunt }\end{array}$ & 8 (13.8\%) & & \\
\hline Choroidal folds & 5 (8.6\%) & & \\
\hline Normal & 7 (12.1\%) & $2(3.8 \%)$ & 5 (8.9\%) \\
\hline
\end{tabular}

Abbreviations: PBT, proton beam therapy; ON, optic nerve.

optic canal opening. Several patients underwent combined procedures. For example, 12 patients had debulking and optic canal opening. Each patient's procedures are detailed in Table 3. One patient had post-PBT surgery because the tumor kept growing post-PBT and contralateral ON was at risk of compression. Two patients had bilateral compressive optic neuropathy. In both cases, patients presented after the left eye had no light perception. These two patients had radiotherapy only for their right compressive optic neuropathy and only data from their right eyes were considered in the analysis.

\section{Eye Examination After Surgery}

Twenty-eight patients had surgery before PBT. Postsurgical eye examination was available in 26. It showed that visual acuity slightly decreased on average postsurgery: it was of 1 logMAR $(\mathrm{IQR}=2.2)$ vs 0.9 ( $\mathrm{IQR}=1.6)$ pre-surgery but this was not statistically significant $(\mathrm{p}=0.27)$. On average, post-surgical visual field MD improved significantly $(-10$ vs $-17, p=0.004$, Mann Whitney), but this includes only patients with the highest visual acuity at presentation, which allowed initial automatized visual field testing.

\section{Proton Beam Therapy}

Total dose delivered was 52.2 GyRBE in 35 patients; 51 GyRBE in 13; 54 GyRBE in 8; 50.4 GyRBE in 1 and 49.3 GyRBE in 1 patient, with dose per fraction of 1.8 GyRBE in 44 patients; 1.7 GyRBE in 14; 2 GyRBE in 1.

Regarding exposure of organs at risk: ipsilateral retina received a mean maximal dose of 47.5 GyRBE (12.1-53.2 GyRBE, median 49.3). Chiasm received a mean maximal 
Table 3 Surgical Procedures

\begin{tabular}{|c|c|c|c|c|c|c|}
\hline \multirow[t]{2}{*}{ Patient } & \multirow{2}{*}{$\begin{array}{c}\text { Biopsy } \\
\text { No Tumorectomy }\end{array}$} & \multicolumn{2}{|c|}{ Debulking } & \multirow[t]{2}{*}{ Orbitotomy } & \multirow[t]{2}{*}{ Optic Nerve Sheath Fenestration } & \multirow[t]{2}{*}{ Optic Canal Opening } \\
\hline & & Orbit & Intracranial & & & \\
\hline I & & & $\mathrm{x}$ & & & $\mathrm{x}$ \\
\hline 3 & & $x$ & & $x$ & & \\
\hline 6 & $x$ & & & & & \\
\hline 11 & $x$ & & & & $x$ & $x$ \\
\hline 14 & & & $x$ & & & $x$ \\
\hline 15 & $x$ & & & & & $x$ \\
\hline 16 & $x$ & & & & & $x$ \\
\hline 21 & & & $x$ & & & $x$ \\
\hline 24 & & $x$ & & & $x$ & $x$ \\
\hline 28 & & & $x$ & $x$ & & $x$ \\
\hline 29 & & & $x$ & & & $x$ \\
\hline 34 & & & $x$ & & $x$ & $x$ \\
\hline 35 & & & $x$ & & $x$ & $x$ \\
\hline 37 & & & & & & \\
\hline 39 & $x$ & & & & $x$ & $x$ \\
\hline 40 & & & & $x$ & & \\
\hline 41 & & & $x$ & & & \\
\hline 42 & & & $\mathrm{x}$ & & $x$ & $x$ \\
\hline 43 & & & $x$ & & $x$ & $x$ \\
\hline 44 & & & $x$ & & & \\
\hline 46 & & & $x$ & & & $x$ \\
\hline 47 & & & $\mathrm{x}$ & & & \\
\hline 48 & & $x$ & & $x$ & & $x$ \\
\hline 49 & $x$ & & & & $x$ & $x$ \\
\hline 51 & & & $\mathrm{x}$ & & & \\
\hline 52 & & $x$ & & & $x$ & $x$ \\
\hline 58 & & & $x$ & & $x$ & $x$ \\
\hline 59 & & $x$ & & & & $x$ \\
\hline 60 & & $x$ & & & $x$ & $x$ \\
\hline TOTAL & 6 & 6 & 15 & 4 & II & 21 \\
\hline
\end{tabular}

dose of 40.6 GyRBE (0.8-52.7 GyRBE, median 50 GyRBE). Those maximal doses reflect the distance between the meningioma extension and respectively the posterior pole of the eye globe and the chiasm.

Mean dose to the ipsilateral eye for 60 patients ranged from 0.5 GyRBE to 31.9 GyRBE (mean 12.9; median 11.7 GyRBE). Mean dose to the lens was available in 38 patients and ranged from 0 to 3.4 GyRBE, mean 0.4 GyRBE, median 0.12. In 2 patients with much larger meningioma (29.3 cc and $33.3 \mathrm{cc}$, respectively) mean dose to the eye globe was 26.3 and 31.9 GyRBE, and mean dose to the lens was 7.9 and 13 GyRBE. These values reflected tumor size, distance from anterior extension of the meningioma to those structures, and the high dose gradient obtained with protons in the eye globe structure (sharp lateral penumbra).
Contralateral optic pathway was protected, with mean maximal dose being 1.15 (median: 0.03 GyRBE) in unilateral meningioma.

Pituitary gland was exposed to mean and median dose of 20.6 and 12.9 GyRBE, ipsilateral temporal lobe 3.6 and 2.8 GyRBE and contralateral temporal lobe 0.51 and 0 GyRBE.

Table 4 Visual Field Changes (55 Patients)

\begin{tabular}{|l|l|l|l|}
\hline & Improved & Stable & Worsened \\
\hline Upfront Surgery + PBT (28) & 13 & 11 & 4 \\
\hline PBT only (3I) & 14 & 9 & 4 \\
\hline Total & 27 & 20 & 8 \\
\hline
\end{tabular}




\section{Eye Examination Pre-PBT and Post-PBT}

Visual acuity pre-PBT was available in 55 patients. It was $0.7 \log$ MAR on average (IQR=1.4). Eighteen patients had visual acuity of $0 \operatorname{logMAR}(20 / 20)$ when they were diagnosed with ONSM. 12 of them did experience progressive visual loss. Thirteen patients had steroid treatment as a first treatment of ON swelling or visual loss. Only 3 of them reported subjective improvement in their vision, but the improvement was temporary and objective measurements did not confirm patients' experience.

Post-PBT examination was performed at least 1 year after the end of radiotherapy. Time span between PBT and final eye examination varied between 1 and 13 years. Median follow-up duration was 48 months (IQR=24-63). No significant changes in visual acuity occurred post-PBT $(0.8 ; \mathrm{IQR}=1.6 ; \mathrm{p}=0.34)$ and the fact of having surgery before PBT did not significantly influence visual outcome post-PBT ( $\mathrm{p}=0.93$, Fisher).

Final visual acuity at last follow-up was significantly correlated to visual acuity pre-PBT ( 0.84 , Spearman). The presence of an opto-ciliary shunt at presentation $(\mathrm{p}=0.03$, Mann Whitney) was significantly associated with decreased vision at presentation and poor prognosis.

Visual field changes are reported in Table 4. Overall, $47 / 55$ (85\%) patients had either improvement or stability in their visual field examination.

\section{MRI Changes}

All 60 patients had an MRI before treatment. For quality reasons, Schick's classification grading was not possible in 2 and adequate measurement of the tumor was impossible in 5 . Nearly $60 \%$ of patients had a tumor that was limited to the orbit (grade I). Fusiform expansion represented the most common presentation of ONSM, found in 35 out of 56 patients. Unsurprisingly, patients with ONSM extension through the optic canal (grade II) and with intracranial extension (grade III) were more likely to undergo surgery and to be classified as a lower grade before PBT. All sixty patients had post-treatment MRI (after PBT alone or associated with surgery). Seven patients changed grade between pre- and post-surgery. Four patients changed grade between pre- and post-PBT ( 2 of them had upfront surgery without grade changes): 2 patients were graded $1 \mathrm{~b}$ post-PBT vs $2 \mathrm{a}$ pre-PBT; 1 patient was graded 1a postPBT vs $1 b$ pre-PBT; 1 patient was graded 2 a post-PBT vs 3a pre-PBT.

\section{Tumor Surface}

As assessed by imaging (WHO criteria detailed previously), 8/55 patients (14\%) were classified as PR, 45/ $55(82 \%)$ as ST, and 2/55 (4\%) as PD. In 47 out of 55 patients $(76 \%)$, tumor surface was stable post-PBT when compared to pre-PBT. In the other 8 patients, tumor surface decreased (14.5\%). No patients had an increase in the surface of the tumor post-PBT. In 2 patients, tumor size increased between first MRI and pre-surgery MRI. In these patients, surgery was scheduled 12 months $(+46 \%$ increase in size) and 85 months $(+203 \%)$ post-PBT.

Three patients had an increase in the size of the tumor between surgery and PBT. In these patients, PBT was performed 18, 26, and 68 months post-surgery. Size of the tumor increased by $27.2 \%, 32.3 \%$, and $111.1 \%$ respectively. Of patients who only had PBT, all exhibited stable tumor size after the procedure, none exhibited significant increase in tumor size, and 8 showed significant shrinking. When comparing the last follow-up MRI to the one performed 1 year post-PBT, we found that tumor size continued to shrink significantly $\left(\mathrm{D} 1 \mathrm{xD} 2^{\text {post } \mathrm{PBT}}=225 ; \mathrm{D} 1 \times \mathrm{xD} 2^{\text {last follow-up }}=212\right.$; $\mathrm{p}=0.0001$ ).

\section{Tolerance and Side Effects}

Excellent early tolerance to PBT was observed. Main reactions were moderate epithelitis, unfrequent limited slight alopecia, pain, and asthenia.

- Radiation optic neuropathy

One patient developed rapidly progressive visual loss over the course of 1 week, 6 months post-PBT. She was diagnosed with ONSM at the age of 23 and was treated with PBT at the age of 51, because of the intracranial extension of the meningioma (grade 3a) and worsening of her visual field. She received a total dose of 54 Gy divided in 30 fractions of $1.8 \mathrm{~Gy}$. After PBT, visual acuity decreased from 0.1 to $2.3 \log$ MAR. Her MRI showed hypersignal of her left optic nerve with Gadolinium enhancement, consistent with RON. Treatment with steroids was considered unsuitable given her history of gastric surgery and heart valve surgery. Follow-up MRI showed disappearance of Gadolinium enhancement at 1 year but without improvement in visual acuity. It is possible that other patients with very poor vision or no light perception also developed toxicity of their ONs related to radiotherapy but we could not detect it due to their severe condition. No lesion 
that could have raised suspicion for RON was seen on follow-up MRIs in any other patients. This complication has previously been reported in an ONSM patient over 2 years post-PBT. ${ }^{12}$

\section{Retinal findings}

Retinal abnormalities were observed in 11 patients during follow-up. Four of them had only PBT, while the other 7 had a combination of surgery and PBT.

\section{Retinal findings in patients with PBT only}

Two patients with exclusive PBT developed mild radiation retinopathy (RR), which manifested as retinal hemorrhages at 15 and 70 months post-PBT. There was no decrease in visual acuity related to these retinal hemorrhages that were discovered at a scheduled follow-up examination. PBT protocol was standard with respective total prescribed doses of 52.2 and 54 GyRBE, dose per fraction of $1.8 \mathrm{GyRBE}$ and maximum dose to the retina of 48 and 52 GyRBE, mean dose to the eye ball 4.3 and 12.7 GyRBE. Visual outcome was excellent in both patients with final visual acuity of $0 \log$ MAR. The third patient with PBT only who developed retinal abnormality had macular edema related to microaneurysm that was treated with anti-VEGF injections in another facility and had no follow-up in our hospital. We were unable to investigate her case further to determine whether this was related to PBT. The last patient showed microcystic degeneration of the retina, related to severe optic nerve atrophy. This last condition is seen in optic nerve atrophy of other etiologies and was, therefore, not considered a side effect of radiotherapy.

\section{Retinal findings in patients with surgery + PBT}

Seven patients, of the combined approach group with upfront surgery, developed retinal manifestations.

Five patients had RR with patchy peripapillar or interpapillomacular (ie, between the optic nerve head and the center of the macula) ischemia at $12,47,53,58$, and 59 months post-PBT. One patient had decrease in visual acuity related to macular ischemia at month 58 but no treatment was deemed necessary because of central location of ischemia and absence of exudation. Visual acuity decreased significantly from $0.4 \operatorname{logMAR}(20 / 50)$ postPBT to $1.3(20 / 400)$ at the last follow-up. In her case, PBT prescribed was 54 GyRBE, dose per fraction 1.8 with maximum dose to the retina of 49 GyRBE, mean dose to the eye ball of 11 GyRBE. In the other four patients, RR was diagnosed upon scheduled follow-up. The patient who developed ischemia at 12 months underwent sectorial retinal photocoagulation, which allowed control of peripheral retinal ischemia and visual acuity remained stable at 0 $\log$ MAR (20/20). In the three last patients, there were few retinal hemorrhages and limited retinal ischemia that caused no changes in visual acuity or visual field and required no treatment. These patients were monitored closely (every 2-4 months) and retinal hemorrhages spontaneously disappeared over time without intervention.

Two patients with no light perception pre-PBT developed macular edema. Of these, one had an epiretinal membrane prior to PBT.

\section{Discussion}

We report here the largest retrospective series of 60 consecutive adult patients with ONSM treated with PBT.

Our results suggest that PBT is a safe and efficient treatment of ONSM either alone or in combination with surgery, but that screening for RR should remain mandatory for several years. They also suggest that the rates of visual improvement, disease control, and side effects are comparable to those reported post-PhRT in the literature.

Combined proton and photon therapies have been used in treatment of skull base tumor and cerebral meningioma. ${ }^{13}$ Few series have reported PBT in ONSM because of limited availability of the procedure, which costs at least twice as much as conventional PhRT. In the largest series previously published by Arvold et al, only 9 patients with ONSM out of 25 were treated with PBT only. ${ }^{14}$ The authors reported stability or improvement in the vision of 7 patients (including one who developed asymptomatic retinopathy), and decreased vision in 1 patient (one last patient was lost to follow-up).

Several series have reported PhRT as an efficient treatment for ONSM with excellent disease control on imaging and visual improvement or stabilization. ${ }^{4}$ Parker et al reviewed various PhRT techniques used between 1999 and 2011: fractionated stereotactic radiotherapy SFRT was used in 19 groups, 3D conformal radiotherapy in 7 groups, and intensity modulated radiotherapy IMRT in 5 groups. These techniques yielded similar results in terms of conservation of vision (stabilization or improvement) obtained in more than $80 \%$ of patients and a $100 \%$ rate of disease control on MRI (stabilization or reduction). ${ }^{4}$ In our series, $85 \%$ of the patients had either 
stability or improvement of their visual field, and $100 \%$ had disease control on MRI, which is comparable to results of PhRT.

The rationale for using PBT is that this technique provides a better dose distribution than PhRT. The finite energy path length of protons allows reduction of dose to surrounding normal tissues, which is a major concern in ONSM given the close relationship between the tumor and the $\mathrm{ON}$, and the proximity of the retina. One would expect lower rate of RR and RON when PBT is used. Unfortunately, this theoretical advantage was not verified clinically, as 7 out of our 60 patients (13\%) developed RR and $1(1.6 \%)$ had RON. The dose of radiotherapy was standard in all these patients. Retrospectively, there were no red flags associated with their cases, except for a big tumor size in the patient with RR and decreased vision in the surgery group. In the literature, RR and RON were reported in $4-18 \%$ of ONSM patients treated with PhRT, with various post-treatment follow-up durations. ${ }^{14-24}$ However, in our series, the rate of retinal complications was significantly higher for patients who underwent upfront surgery. In the surgery group, $17 \%$ of patients developed RR, as opposed to $6 \%$ in the PBT only group. Higher likelihood to develop RR after a combined approach has also been reported in patients treated with PhRT. $^{25}$

Depending on the location and size of the tumor, ONSM can lead to poor vision and blindness. ${ }^{1}$ Schick et al reported a large series of ONSM patients who underwent surgery. They found a correlation between visual loss and duration of the disease before treatment and, consequently, recommended radiotherapy for intraorbital tumors. ${ }^{26}$ Rassi et al reported that patients with good preoperative vision and CSF flow in the optic sheath had better chances of a favorable outcome than those with poor vision. $^{27}$

In our series, ONSM was diagnosed on average 16 months after patients first noticed changes in their vision or reported symptoms related to ONSM. There was no difference in delay in diagnosis between patients with or without documented visual acuity. No specific ONSMrelated symptoms or specific fundus abnormalities were linked to shorter or longer diagnostic delay. Even though no specific symptoms or signs were significantly correlated with delay, it is likely that factors such as the rarity of the condition, insidious initial involvement, and late presentation explain why diagnosis was not made sooner. For instance, compressive optic neuropathy can mimic normal-tension glaucoma, which is significantly more common. This can be misleading for comprehensive ophthalmologists. Although most patients $(86.7 \%$ in our study) reported visual loss, the latter was often progressive. Patients might not have sought medical advice as quickly as they would have in case of a sudden decrease in vision. Furthermore, in $12 \%$ of our patients fundus examination at presentation was normal. This could have misled ophthalmologists in the early stages since visual field damage was subtle. Eighteen patients had visual acuity of $0 \operatorname{logMAR}(20 / 20)$ when they were diagnosed with ONSM and 12 of them did experience progressive visual loss. This was explained by centralsparing visual field defect. There was indeed discrepancy between visual acuity and mean deviation in the visual field.

There is no pathognomonic sign of ONSM. Fundus examination can show edema, pallor or cupping of the ON, which are seen in many other optic neuropathies. In our series, about half of the patients $(46.5 \%)$ presented with optic disc swelling and almost all of them developed pallor over time, independently of surgery. $84.4 \%$ of the patients showed either swelling or pallor of ON head at presentation. Pallor of the optic disc at presentation was not significantly related to the involvement of the optic canal $(50 \%$ of the patients, with or without optic disc pallor, had a tumor grade of 2 or 3 by Schick classification at presentation). However, patients with optic disc swelling were more likely to have a bigger sized tumor with optic canal or intracranial involvement ( $61.5 \%$ vs $40 \%)$.

Opto-ciliary shunts are a sign of compression of the $\mathrm{ON}$ and congestion of the central retinal vein. ${ }^{28}$ Their presence was associated with poor prognosis, as 6 out of 8 patients with shunts had final vision of less than 0.6 $\log$ MAR (20/200). Patients with opto-ciliary shunts had significantly worse visual outcome than other patients, regardless of the type of treatment. Five patients had choroidal folds and, in 2 of them, it was the only fundus ophthalmoscopy finding. Choroidal folds are consistent with retrobulbar lesion exerting mass effect on the sclera.

There were no significant changes in visual acuity and visual field testing pre-PBT or at 1 year post-PBT. The number of patients with greater improvement in visual acuity ( $>0.4 \log$ MAR) was higher in the surgery group. This improvement was found post-surgery and visual acuity was then found stable post-PBT. Surgery plays a role in relieving compression on the ON, which allows rapid recovery of a suffering ON. On the other hand, 
radiotherapy has shown efficacy at stabilizing the tumor size and at preventing further worsening of vision, when measured in terms of visual acuity. When visual field is considered, a majority of patients show improvement.

In our series, it is worth mentioning that no patient died after surgery. Sealing is challenging to obtain in meningioma surgery despite careful dural reconstruction and use of biological glue. Morbidity consisted of local inflammation and subcutaneous cerebrospinal fluid extravasation, which resolved either spontaneously or after repeat lumbar puncture or compression bandage.

The current study suffers from the usual flaws of retrospective investigations. Even though patient follow-up is standardized in our institution, patients were seen several years apart and timing for post-treatment examination varied greatly between patients. Several data were missing, including initial visual field examination and RNFL measurement that were not systematically performed before 2005. RR can be a late complication of PBT, as shown in our results with patients developing visual loss from retinal ischemia 5 years after the end of treatment, and not all of the patients in this study were followed for such a long time period. It is possible, then, that the number of these complications is underestimated. However, this side effect of PBT can occur without visual disturbances. Patients should therefore be monitored closely and referred to a retina specialist as soon as suspicion for retinopathy is raised on the basis of fundus examination. Our patient with RR who needed PPR had had surgery. One can hypothesize that surgery had weakened the vascular network of the retina. We recommend that the fundus of patients who received surgery and PBT should be monitored more closely and for longer than patients who had only PBT.

\section{Conclusion}

From our experience, PBT appears to be a safe and efficient treatment for primary ONSM. It controls tumor growth and in some cases even reduces it. It significantly improves visual field, with visual acuity remaining stable in most cases. Presence of an opto-ciliary shunt, related to chronic optic disc ischemia, had a bad prognosis. Surgery remains helpful in complicated cases and was efficient at reducing visual loss related to compression at the optic canal. There were no acute side effects of PBT on visual function but late radiation retinopathy can occur and requires regular monitoring of fundus examination up to 6 years post-PBT. Overall, our results suggest that $\mathrm{PhRT}$ and $\mathrm{PBT}$ are comparable from a clinical standpoint. Both seem to have similar outcomes and both produce irradiation effects. This should raise questions about the desirability of PBT as a routine treatment for ONSM, especially in light of the well-documented financial burden it imposes on medical institutions.

\section{Data Sharing Statement}

Yes.

\section{Ethical Approval}

All procedures performed in studies involving human participants were in accordance with the ethical standards of the institutional and/or national research committee and with the 1964 Helsinki declaration and its later amendments or comparable ethical standards.

\section{Informed Consent}

All individual participants were informed and gave their consent for the use of their data in this retrospective study.

\section{Acknowledgments}

The authors would like to thank David Peña-Guzmán, $\mathrm{PhD}$, for his advice and contribution in finalizing the manuscript.

\section{Funding}

There is no funding to report.

\section{Disclosure}

All authors report no conflicts of interest related to this study.

\section{References}

1. Douglas VP, Douglas KAA, Cestari DM. Optic nerve sheath meningioma. Curr Opin Ophthalmol. 2020;31:455-461. doi:10.1097/ ICU.0000000000000700

2. Hénaux P-L, Bretonnier M, Le Reste P-J, Morandi X. Modern management of meningiomas compressing the optic nerve: a systematic review. World Neurosurg. 2018;118:e677-86. doi:10.1016/j. wneu.2018.07.020

3. Narayan S, Cornblath WT, Sandler HM, Elner V, Hayman JA. Preliminary visual outcomes after three-dimensional conformal radiation therapy for optic nerve sheath meningioma. Int $J$ Radiat Oncol Biol Phys. 2003;56:537-543. doi:10.1016/S0360-3016(03)00005-1

4. Parker RT, Ovens CA, Fraser CL, Samarawickrama C. Optic nerve sheath meningiomas: prevalence, impact, and management strategies. Eye Brain. 2018;10:85-99. doi:10.2147/EB.S144345

5. Paulsen F, Doerr S, Wilhelm H, Becker G, Bamberg M, Classen J. Fractionated stereotactic radiotherapy in patients with optic nerve sheath meningioma. Int J Radiat Oncol Biol Phys. 2012;82:773-778. doi:10.1016/j.ijrobp.2010.11.018

6. Pitz S, Becker G, Schiefer U, et al. Stereotactic fractionated irradiation of optic nerve sheath meningioma: a new treatment alternative. $\mathrm{Br}$ J Ophthalmol. 2002;86:1265-1268. doi:10.1136/bjo.86.11.1265 
7. Shrieve DC, Hazard L, Boucher K, Jensen RL. Dose fractionation in stereotactic radiotherapy for parasellar meningiomas: radiobiological considerations of efficacy and optic nerve tolerance. J Neurosurg. 2004;101(Suppl 3):390-395. doi:10.3171/sup.2004.101 supplement3.0390

8. Danesh-Meyer HV. Radiation-induced optic neuropathy. J Clin Neurosci. 2008;15:95-100.

9. Schick U, Dott U, Hassler W. Surgical management of meningiomas involving the optic nerve sheath. J Neurosurg. 2004;101:951-959. doi:10.3171/jns.2004.101.6.0951

10. Palmer MK. WHO handbook for reporting results of cancer treatment. $\mathrm{Br} J$ Cancer. 1982;45(3):484-485. doi:10.1038/ bjc. 1982.83

11. Kahraman-Koytak P, Bruce BB, Peragallo JH, Newman NJ, Biousse V. Diagnostic errors in initial misdiagnosis of optic nerve sheath meningiomas. JAMA Neurol. 2019;76:326-332. doi:10.1001/ jamaneurol.2018.3989

12. Siddiqui JD, Loeffler JS, Murphy MA. Radiation optic neuropathy after proton beam therapy for optic nerve sheath meningioma. J Neuro-Ophthalmol. 2013;33:165-168. doi:10.1097/ WNO.0b013e31828292b8

13. Wenkel E, Thornton AF, Finkelstein D, et al. Benign meningioma: partially resected, biopsied, and recurrent intracranial tumors treated with combined proton and photon radiotherapy. Int $J$ Radiat Oncol Biol Phys. 2000;48:1363-1370. doi:10.1016/S0360-3016(00)01411-5

14. Arvold ND, Lessell S, Bussiere M, et al. Visual outcome and tumor control after conformal radiotherapy for patients with optic nerve sheath meningioma. Int $J$ Radiat Oncol Biol Phys. 2009;75:1166-1172. doi:10.1016/j.ijrobp.2008.12.056

15. Eckert F, Clasen K, Kelbsch C, et al. Retrospective analysis of fractionated intensity-modulated radiotherapy (IMRT) in the interdisciplinary management of primary optic nerve sheath meningiomas. Radiat Oncol Lond Engl. 2019;14:240. doi:10.1186/s13014-0191438-2

16. Ratnayake G, Oh T, Mehta R, et al. Long-term treatment outcomes of patients with primary optic nerve sheath meningioma treated with stereotactic radiotherapy. J Clin Neurosci. 2019;68:162-167.

17. Pandit R, Paris L, Rudich DS, Lesser RL, Kupersmith MJ, Miller NR. Long-term efficacy of fractionated conformal radiotherapy for the management of primary optic nerve sheath meningioma. Br J Ophthalmol. 2019;103:1436-1440. doi:10.1136/bjophthalmol2018-313135

18. Jin J, Joo JD, Han JH, et al. Optic nerve sheath meningioma: preliminary analysis of the role of radiation therapy. Brain Tumor Res Treat. 2018;6:8-12. doi:10.14791/btrt.2018.6.e2
19. Soldà F, Wharram B, Gunapala R, Brada M. Fractionated stereotactic conformal radiotherapy for optic nerve sheath meningiomas. Clin Oncol $R \quad$ Coll Radiol. 2012;24:e106-112. doi:10.1016/j. clon.2012.03.015

20. Abouaf L, Girard N, Lefort T, et al. Standard-fractionated radiotherapy for optic nerve sheath meningioma: visual outcome is predicted by mean eye dose. Int $J$ Radiat Oncol Biol Phys. 2012;82:1268-1277. doi:10.1016/j.ijrobp.2011.04.010

21. Saeed $P$, Blank L, Selva D, et al. Primary radiotherapy in progressive optic nerve sheath meningiomas: a long-term follow-up study. $\mathrm{Br}$ J Ophthalmol. 2010;94:564-568. doi:10.1136/bjo.2009.166793

22. Metellus P, Kapoor S, Kharkar S, et al. Fractionated conformal radiotherapy for management of optic nerve sheath meningiomas: long-term outcomes of tumor control and visual function at a single institution. Int $J$ Radiat Oncol Biol Phys. 2011;80:185-192. doi:10.1016/j.ijrobp.2010.01.034

23. Andrews DW, Faroozan R, Yang BP, et al. Fractionated stereotactic radiotherapy for the treatment of optic nerve sheath meningiomas: preliminary observations of 33 optic nerves in 30 patients with historical comparison to observation with or without prior surgery. Neurosurgery. 2002;51:890-902; discussion 903-904.

24. Baumert BG, Villà S, Studer G, et al. Early improvements in vision after fractionated stereotactic radiotherapy for primary optic nerve sheath meningioma. Radiother Oncol J Eur Soc Ther Radiol Oncol. 2004;72:169-174. doi:10.1016/j.radonc.2004.04.008

25. Turbin RE, Thompson CR, Kennerdell JS, Cockerham KP, Kupersmith MJ. A long-term visual outcome comparison in patients with optic nerve sheath meningioma managed with observation, surgery, radiotherapy, or surgery and radiotherapy. Ophthalmology. 2002;109:890-9; discussion 899-900. doi:10.1016/S0161-6420(02) 01017-5

26. Schick U, Jung C, Hassler WE. Primary optic nerve sheath meningiomas: a follow-up study. Cent Eur Neurosurg. 2010;71:126-133. doi:10.1055/s-0029-1246136

27. Rassi MS, Prasad S, Can A, Pravdenkova S, Almefty R, Al-Mefty O. Prognostic factors in the surgical treatment of intracanalicular primary optic nerve sheath meningiomas. J Neurosurg. 2018;1-8. doi:10.3171/2018.4.JNS173080

28. Dutton JJ. Optic nerve sheath meningiomas. Surv Ophthalmol. 1992;37:167-183. doi:10.1016/0039-6257(92)90135-G
Eye and Brain

\section{Publish your work in this journal}

Eye and Brain is an international, peer-reviewed, open access journal focusing on clinical and experimental research in the field of neuroophthalmology. All aspects of patient care are addressed within the journal as well as basic research. Papers covering original research, basic science, clinical and epidemiological studies, reviews and

Submit your manuscript here: https://www.dovepress.com/eye-and-brain-journal evaluations, guidelines, expert opinion and commentary, case reports and extended reports are welcome. The manuscript management system is completely online and includes a very quick and fair peerreview system, which is all easy to use. Visit http://www.dovepress. com/testimonials.php to read real quotes from published authors. 